\title{
Resting-State Functional Connectivity in Patients with Long-Term Remission of Cushing's Disease
}

\author{
Steven JA van der Werff ${ }^{*, 1,2}$, J Nienke Pannekoek ${ }^{1,2}$, Cornelie D Andela ${ }^{2,3}$, Onno C Meijer ${ }^{2,3}$, \\ Mark A van Buchem ${ }^{2,4}$, Serge ARB Rombouts ${ }^{2,4,5}$, Roos C van der Mast', Nienke R Biermasz ${ }^{2,3}$, \\ Alberto M Pereira ${ }^{2,3}$ and Nic JA van der Wee Wen $^{1,2}$ \\ 'Department of Psychiatry, Leiden University Medical Center, Leiden, The Netherlands; ' Leiden Institute for Brain and Cognition, Leiden, \\ The Netherlands; ${ }^{3}$ Department of Endocrinology and Metabolic Diseases and Center for Endocrine Tumors, Leiden University Medical Center, \\ Leiden, The Netherlands; ${ }^{4}$ Department of Radiology, Leiden University Medical Center, Leiden, The Netherlands; ${ }^{5}$ Institute of Psychology, \\ Leiden University, Leiden, The Netherlands
}

\begin{abstract}
Glucocorticoid disturbance can be a cause of psychiatric symptoms. Cushing's disease represents a unique model for examining the effects of prolonged exposure to high levels of endogenous cortisol on the human brain as well as for examining the relation between these effects and psychiatric symptomatology. This study aimed to investigate resting-state functional connectivity (RSFC) of the limbic network, the default mode network (DMN), and the executive control network in patients with long-term remission of Cushing's disease. RSFC of these three networks of interest was compared between patients in remission of Cushing's disease $(n=24 ; 4$ male, mean age $=44.96$ years) and matched healthy controls ( $n=24 ; 4$ male, mean age $=46.5$ years), using probabilistic independent component analysis to extract the networks and a dual regression method to compare both groups. Psychological and cognitive functioning was assessed with validated questionnaires and interviews. In comparison with controls, patients with remission of Cushing's disease showed an increased RSFC between the limbic network and the subgenual subregion of the anterior cingulate cortex (ACC) as well as an increased RSFC of the DMN in the left lateral occipital cortex. However, these findings were not associated with psychiatric symptoms in the patient group. Our data indicate that previous exposure to hypercortisolism is related to persisting changes in brain function.

Neuropsychopharmacology (2015) 40, I888-1898; doi:I0.1038/npp.20I5.38; published online 25 February 2015
\end{abstract}

\section{INTRODUCTION}

In various stress-related psychiatric disorders such as major depressive disorder and posttraumatic stress disorder (PTSD), alterations in the activity of the hypothalamicpituitary-adrenal axis (HPA axis) are present, with hyperresponsivity or chronic activation of the HPA axis often resulting in increased levels of cortisol (Carroll et al, 1976, 2007; Friedman et al, 2007; Inslicht et al, 2006; Schlechte et al, 1986; Steudte et al, 2011; Young and Breslau, 2004). Animals chronically exposed to increased levels of corticosterone display an anxiodepressive-like phenotype, providing a neuroendocrine animal model for stress-related disorders (Darcet et al, 2014; David et al, 2009). However, extrapolation of findings of this animal model to humans may not always be appropriate, for example when studying human brain activation patterns during specific challenges or resting state.

*Correspondence: SJA van der Werff, Department of Psychiatry, Leiden University Medical Center, PO Box 9600, 2300 RC Leiden, The Netherlands, Tel: +31 71526 228I, Fax: +31 715248156 , E-mail: S.J.A.van_der_Werff@lumc.nl

Received 30 September 2014; revised 8 January 2015; accepted 9 January 2015; accepted article preview online 5 February 2015
Cushing's disease represents a unique human model for examining the effects of prolonged exposure to increased levels of endogenous cortisol on brain structure and function, and the relation between these effects and psychiatric symptomatology. In Cushing's disease, hypercortisolism is caused by an adrenocorticotropic hormone (ACTH) producing pituitary adenoma, stimulating the adrenal glands to continuously release cortisol. In patients with Cushing's disease, a variety of psychiatric symptoms can be induced by hypercortisolism. Major depressive disorder is the most common psychiatric disorder seen in Cushing's disease patients, but comorbidity also includes anxiety, mania, and cognitive dysfunction (Sonino and Fava, 2001). The majority of these symptoms are also seen in stress-related psychiatric disorders, suggesting a common underlying mechanism. After successful biochemical treatment of Cushing's disease, psychiatric symptoms decrease, but patients still show cognitive impairment (Dorn and Cerrone, 2000; Forget et al, 2002; Merke et al, 2005; Resmini et al, 2012; Tiemensma et al, 2010b), decreased quality of life (Tiemensma et al, 2011), and a higher prevalence of affective disorders and apathy (Tiemensma et al, 2010a) compared with healthy controls. These findings suggest that hypercortisolism may cause persisting changes in the brain. 
Effects of hypercortisolism on brain structure include a reduction of hippocampal volume (Bourdeau et al, 2002; Simmons et al, 2000; Starkman et al, 1992) that is reversible after successful abrogation of the hypercortisolism (Bourdeau et al, 2002; Starkman et al, 1999). In addition, our group found reduced anterior cingulate cortex (ACC) gray matter volumes and increased gray matter volumes in the cerebellum in patients with remission of Cushing's disease (Andela et al, 2013), as well as widespread reductions of white matter integrity (van der Werff et al, 2014). Remarkably, similar patterns of reduced hippocampal volume (Bremner et al, 2003; Campbell et al, 2004; Colla et al, 2007; Gurvits et al, 1996; Kitayama et al, 2005; Videbech and Ravnkilde, 2004), reversibility of hippocampal atrophy after treatment (Boldrini et al, 2013; Nordanskog et al, 2010; Tendolkar et al, 2013; Vermetten et al, 2003), and reductions of ACC volumes (Kasai et al, 2008; Thomaes et al, 2010; Yamasue et al, 2003) have been found in stress-related psychiatric disorders.

The effects of hypercortisolism on brain functional connectivity have yet to be investigated. One important network that should be investigated in light of hypercortisolism is the limbic network, consisting of the hypothalamus, hippocampus, amygdala, insula, and parts of the nucleus accumbens (Janes et al, 2012; Maclean, 1952). This network is involved in emotional processing and regulation, as well as the encoding of memories. In addition, the components of this network are responsible for the initial stress response and activation of the HPA axis, with subsequent release of corticotropin-releasing hormone (CRH), followed by ACTH that, in turn, stimulates the adrenal glands to secrete cortisol (Shin and Liberzon, 2010). Exposure to severe stress has been related to altered functional connectivity of various components of the limbic network (Admon et al, 2009; Brown et al, 2014; Jin et al, 2014). The network has consistently been found in other resting-state analysis using an ICA approach (Janes et al, 2012; Laird et al, 2011; Veer et al, 2010). However, naming of the network has been less consistent, with studies using the terms limbic network (Janes et al, 2012), medial temporal network (Laird et al, 2011; Veer et al, 2010), and amygdala-hippocampus complex (Veer et al, 2010) to indicate the same network. Another network of interest is the default mode network (DMN). This network consists of the precuneus, the posterior cingulate cortex, the medial prefrontal cortex, and parts of the parietal cortex, and is involved in the retrieval and manipulation of episodic memories and semantic knowledge, self-referential processing, and prospective memory (Fox et al, 2005; Raichle et al, 2001). Alterations in DMN connectivity have been found in stress-related disorders (Bluhm et al, 2009a, b; Zhu et al, 2012). A third network of interest is the executive control network, consisting of the bilateral dorsolateral prefrontal cortex (PFC), ventrolateral PFC, dorsomedial PFC, and lateral parietal cortices (Beckmann et al, 2005; Seeley et al, 2007). This network is involved in attention demands, working memory, and cognitive control, and is relevant because of the cognitive impairment in patients with long-term remission of Cushing's disease.

The aim of this study was to examine alterations in resting-state functional connectivity (RSFC) in patients with long-term remitted Cushing's disease. We anticipated to find aberrant RSFC with the limbic network, the DMN, and the executive control network in these patients compared with matched healthy controls.

\section{MATERIALS AND METHODS}

\section{Sample Description}

All patients with long-term remission of Cushing's disease monitored in a unique cohort at the Leiden University Medical Center $(n=49)$ and between 18 and 60 years of age were invited by letter and those who did not respond were contacted by phone. The response rate was $96 \%$. Thirty-one patients were willing to participate and were screened for eligibility. Six patients were excluded because of one of the following exclusion criteria: a (history of) drug or alcohol abuse, neurological problems, contraindications for undergoing a magnetic resonance imaging (MRI) scan and left-handedness. Healthy controls were pair-wise matched to the patients based on gender, age, and education and recruited by advertisements in grocery stores and via Internet. In addition to the general exclusion criteria of the study, a history or presence of a psychiatric disorder was an exclusion criterion for the control group. At the day of the MRI scan, patients were asked again about a lifetime history of psychiatric disorders. One patient reported a history of major depressive disorder before the suspected onset of the Cushing's disease.

A total of 25 patients with remission of Cushing's disease and 25 matched healthy controls were included in this study. The MRI scanning session of one patient was aborted prematurely before the resting-state scan was acquired. Therefore, we excluded data from this patient and the matched healthy control from the current study, resulting in a total sample size of 24 patients and 24 controls. The diagnosis of active Cushing's disease had been confirmed in all patients, following previously described criteria (Tiemensma et al, 2010b). All patients were treated with transsphenoidal surgery. Thirteen patients remained glucocorticoid dependent after surgery and were substituted with hydrocortisone (on average $20 \mathrm{mg} /$ day, divided into three dosages). Remission of the Cushing's disease was confirmed in the 11 other patients. The estimated duration of disease was determined through patients' history by looking for the earliest physical/somatic signs. Duration of remission was calculated from the date of curative transsphenoidal surgery, or in case of persistent disease, from the date of normalization of biochemical tests after postoperative radiotherapy. Demographics and patient characteristics are reported in Table 1. Written informed consent was obtained from all participants before the clinical assessment and the MRI scan session. The medical ethical committee of the Leiden University Medical Center approved the study protocol.

\section{Clinical Data Acquisition}

Presence and severity of depressive symptoms were evaluated using the Montgomery-Åsberg depression rating scale (MADRS) (Montgomery and Asberg, 1979) and the Inventory of Depression Symptomatology (IDS) (Rush et al, 1996). Anxiety was evaluated using the Beck Anxiety Inventory (BAI) (Beck et al, 1988) and the Fear Questionnaire (FQ) (Marks and Mathews, 1979). Apathy and irritability were assessed using the Apathy Scale (AS) (Starkstein et al, 2001) 
Table I Demographics of the Total Sample and Clinical Characteristics of the Patients with Long-Term Remission of Cushing's Disease

\begin{tabular}{|c|c|c|}
\hline Characteristics & $\begin{array}{l}\text { Patients with } \\
\text { long-term remission } \\
\text { of Cushing's } \\
\text { disease }(n=24)\end{array}$ & $\begin{array}{c}\text { Matched } \\
\text { healthy } \\
\text { controls } \\
(n=24)\end{array}$ \\
\hline Gender (male/female) & $4 / 20$ & $4 / 20$ \\
\hline Age (years), mean (SD) & $44.96(7.52)$ & $46.5(7.06)$ \\
\hline \multicolumn{3}{|l|}{ Education, n (\%) } \\
\hline Low & $6(25 \%)$ & $6(25 \%)$ \\
\hline Intermediate & II (45.8\%) & $10(41.7 \%)$ \\
\hline High & $7(29.2)$ & $8(33.3 \%)$ \\
\hline \multicolumn{3}{|l|}{ Surgery, n (\%) } \\
\hline Transsphenoidal adenomectomy & $24(100 \%)$ & \\
\hline Bilateral adrenalectomy & $2(8.3 \%)$ & \\
\hline Radiotherapy, n (\%) & $6(25 \%)$ & \\
\hline Disease duration (years), mean (SD) & $8.30(8.12)$ & \\
\hline Duration of remission (years), mean (SD) & $10.92(8.36)$ & \\
\hline \multicolumn{3}{|l|}{ Hypopituitarism, n (\%) } \\
\hline Any axis & $14(58.3 \%)$ & \\
\hline $\mathrm{GH}$ & $10(41.7 \%)$ & \\
\hline $\mathrm{LH} / \mathrm{FSH}$ & $9(37.5 \%)$ & \\
\hline $\mathrm{TSH}$ & $10(41.7 \%)$ & \\
\hline $\mathrm{ADH}$ & $3(12.5 \%)$ & \\
\hline Hydrocortisone substitution & $13(54.2 \%)$ & \\
\hline \multicolumn{3}{|l|}{ Clinical severity index (CSI), mean (SD) } \\
\hline Active phase, total & $8.08(2.02)$ & \\
\hline Remission phase, total & $2.50(1.53)$ & \\
\hline
\end{tabular}

and the Irritability Scale (IS) (Chatterjee et al, 2005), respectively. The Cognitive Failures Questionnaire (CFQ) (Broadbent et al, 1982) was used to assess cognitive functioning. The Cushing's syndrome Severity Index (CSI) (Sonino et al, 2000) was used to assess current severity of symptoms and to retrospectively estimate (clinical) severity at the time of active disease.

The MADRS was assessed before scanning on the scanning day. For all other questionnaires, the time between the scan and filling out the questionnaires was on average $1.4 \pm$ 3.2 days (range 0-19). This broad range was mainly caused by one outlier of 19 days, whereas all other participants filled out questionnaires within 1 week. Moreover, $83 \%$ of the participants filled out questionnaires within 1 day after scanning.

\section{MRI Data Acquisition}

Imaging data were acquired on a Philips 3T magnetic resonance imaging system (software version 3.2.1; Philips Healthcare, Best, The Netherlands). A SENSE-32 channel head coil was used for radio frequency transmission and reception. Beforehand, the participants were instructed to lie as still as possible, with their eyes closed and without falling asleep. After completion of the scan, all participants confirmed not having fallen asleep. Resting-state fMRI (RSfMRI) data were acquired using $\mathrm{T} 2^{*}$-weighted gradient-echo echo-planar imaging with the following scan parameters: 200 whole-brain volumes, repetition time $(\mathrm{TR})=2200 \mathrm{~ms}$, echo time $(\mathrm{TE})=30 \mathrm{~ms}$, flip angle $=80^{\circ}, 38$ axial slices, matrix size $=80 \times 80$, voxel size $=2.75 \times 2.75 \times 2.75 \mathrm{~mm}$, slice gap $=$ 0.275 , scan duration $=449 \mathrm{~s}$.

A high-resolution anatomical image (T1-weighted sequence; $\mathrm{TR}=9.8 \mathrm{~ms}$, $\mathrm{TE}=4.6 \mathrm{~ms}, 140$ axial slices, matrix size $=256 \times 256$, voxel size $1.17 \times 1.17 \times 1.2 \mathrm{~mm}$, no slice gap, scan duration $=296 \mathrm{~s}$ ), and a high-resolution $\mathrm{T} 2{ }^{*}$-weighted gradient echo EPI scan $(T R=2200 \mathrm{~ms}$, TE $=30 \mathrm{~ms}$, flip angle $=80^{\circ}, 84$ axial slices, matrix size $=112 \times 112$, voxel size $=1.96 \times 1.96 \times 2 \mathrm{~mm}$, no slice gap, scan duration $=46.2 \mathrm{~s}$ ) were acquired for registration to standard space.

A neuroradiologist, blinded to the status of the subjects, examined all anatomical images. Apart from incidental agerelated white matter hyperintensities and minor effects of the posttranssphenoidal surgery in the perisellar area, no other macroscopic abnormalities were observed in the patients and controls.

\section{Data Preprocessing}

All MRI data were processed using FSL (FMRIB's Software Library, www.fmrib.ox.ac.uk/fsl; version 5.0.6; Smith et al, 2004). Non-brain tissue removal was applied to the highresolution T1-weighted image and the high-resolution EPI image. The preprocessing of the RS-fMRI was carried out using FMRI Expert Analysis Tool (FEAT). Motion correction was applied to the RS-fMRI data along with non-brain tissue removal, spatial smoothing using a 6-mm full-width at halfmaximum (FWHM) Gaussian kernel, grand-mean intensity normalization of the entire $4 \mathrm{D}$ data set by a single multiplicative factor and high-pass temporal filtering (Gaussianweighted least-squares straight line fitting, $0.01 \mathrm{~Hz}$ cutoff).

The RS-fMRI data of each participant were then registered to their respective high-resolution EPI images. The highresolution EPI image was registered to the T1-weighted image, and the T1-weighted image to MNI-152 standard space image (T1-weighted standard brain averaged over 152 subjects; Montreal Neurological Institute, Montreal, QC, Canada), with a resampled resolution of $4 \mathrm{~mm}$.

\section{Extracting Resting-State Networks}

Analysis was carried out using Probabilistic Independent Component Analysis (PICA; Beckmann and Smith, 2004) as implemented in MELODIC (Multivariate Exploratory Linear Decomposition into Independent Components). We opted to use this method over a seed-based approach, because our hypotheses were aimed at investigating connectivity with networks rather than with more localized areas. In addition, PICA gives us the advantage to distinguish physiological signals from noise, as they are separated in different components. This technique also enables us to omit global signal regression that is known to induce negative connectivity between networks (Murphy et al, 2009). Default group PICA 
processing steps were applied to the individual preprocessed and normalized data sets: masking of non-brain voxels, voxel-wise de-meaning of the data, and normalization of the voxel-wise variance based on all data sets. Subsequently, the preprocessed data were concatenated in time to create a single $4 \mathrm{D}$ data set that was then projected into a 20 -dimensional subspace using principal component analysis. The observations were decomposed into 20 sets of independent vectors that describe signal variation across the temporal (time courses) and spatial (maps) domains by optimizing for non-Gaussian spatial source distributions using a fixed-point iteration technique (Hyvarinen, 1999). We chose to use 20 independent components to reach the same balance between the amount of clustering and splitting as previous studies applying the same techniques (Smith et al, 2009a). The resulting estimated component maps were divided by the SD of the residual noise and thresholded at a probability threshold of $p>0.5$ (ie, an equal weight is placed on false positives and false negatives) by fitting a mixture model to the histogram of intensity values.

\section{Statistical Analyses}

The set of spatial maps from the group-average analysis was used to generate subject-specific versions of the spatial maps, and associated time courses, using dual regression (Filippini et al, 2009). In short, for each subject, the group-average set of spatial maps was regressed as spatial regressors in a multiple regression into the preprocessed individual $4 \mathrm{D}$ resampled data set. This resulted in a subject-specific time course for each component separately. Next, those time courses were regressed as temporal regressors in a multiple regression into the same preprocessed individual $4 \mathrm{D}$ data set, resulting in a set of subject-specific spatial maps, one for each of the 20 components. In addition, subject-specific $z$-maps for each of the components were constructed through normalization of the subject-specific spatial maps by the residual within-subject noise.

Upon visual inspection, we selected three components based on spatial similarity to functional networks described before: the limbic network (Shin and Liberzon, 2010), the DMN (Beckmann et al, 2005; Fox et al, 2005; Raichle et al, 2001), and the executive control network (Seeley et al, 2007). We segmented the $1 \mathrm{~mm}$ MNI-152 standard brain into gray matter, white matter, and cerebrospinal fluid (CSF) and created a mask comprising gray and white matter only. This mask was then registered to an isotropic resolution of $4 \mathrm{~mm}$ and applied to the study-specific mask in order to exclude voxels containing CSF from our analyses.

The subject-specific spatial maps of each of these three components along with the study-specific gray and white matter mask were fed into FSL's Randomise tool. The groups were compared using a general linear model (GLM) including age and level of education as confound regressors. In addition, for each subject, gray matter density maps were derived from the anatomical scans using FSL. Previously, Cushing's disease-related gray matter abnormalities were reported in the same sample (Andela et al, 2013). Therefore, to control for structural abnormalities possibly confounding differences in functional connectivity and to correct for the effects of possible misregistration (Oakes et al, 2007), information about gray matter values of each subject was included as a voxel-wise confound regressor in the GLM. Between- and within-group effects were tested using permutation-based (5000 permutations) nonparametric testing. To control for family-wise error we applied threshold-free cluster enhancement (TFCE; (Smith and Nichols, 2009b), and statistical maps were thresholded at $p<0.05$.

\section{RESULTS}

\section{Psychopathology Scores}

The psychometric data are reported in Table 2. To correct for multiple comparisons we applied a Bonferroni correction and adjusted the level of significance to $p<0.005$. Comparisons of the psychometric data between patients with longterm remission of Cushing's disease and the matched healthy controls showed significant differences on the MADRS $(p<0.001)$ and AS $(p=0.001)$. The following psychometric data showed uncorrected differences between groups: IDS $(p=0.008)$, BAI $(p=0.010)$, CFQ $(p=0.028)$, and FQ $(p=$ $0.006)$. The only subscale of the FQ that showed an uncorrected difference between groups was the social phobia subscale $(p=0.012)$.

\section{Resting-State Functional Connectivity}

Motion parameters (Table 2) did not differ significantly between groups for both absolute displacement $(t=-1.017$, $p=0.315)$ and relative displacement $(t=0.355, p=0.725)$. The three networks of interest were identified among the 20 components resulting from the PICA (Figure 1). These three networks have been consistently present across subjects and over time using PICA in previous studies (Beckmann et al, 2005; Veer et al, 2010). All three functional networks were present in both the patient group and healthy control group. Between-group differences in RSFC for each network were examined by comparing the following contrasts: (1) controls $<$ patients and (2) controls $>$ patients. These comparisons revealed a between-group difference in RSFC between the limbic network and the subgenual subregion of the ACC $(p<0.05$, TFCE corrected; Figure 2a). To determine whether this effect was driven by a decrease in negative connectivity or an increase in positive connectivity, mean individual $z$-scores for this region were extracted from the subjectspecific $z$-maps of the limbic network component. Comparison of the mean $z$-scores of both groups showed an increase in positive connectivity of the limbic network with the subgenual ACC in patients with Cushing's disease in remission compared with healthy controls (Figure 2b). To determine whether the strength of RSFC between the limbic network and the subgenual ACC was associated with psychopathology scores of the patients with long-term remission of Cushing's disease, we performed linear regression analyses with adjustment for age and education. The psychopathology scores reported in Table 2, as well as the active and the remission subscales of the CSI, and the disease and remission duration in years did not show a significant association with the strength of the RSFC between the limbic network and the subgenual ACC in patients with long-term remission of Cushing's disease (for all: $p>0.1$ ). Furthermore, the found effect could not be related to treatment with hydrocortisone as there was no significant difference in $z$-scores between the 
Table 2 Symptom Severity Scores of Patients with Long-Term Remission of Cushing's Disease Versus Matched Healthy Controls

\begin{tabular}{|c|c|c|c|}
\hline & $\begin{array}{l}\text { Patients with long-term remission } \\
\text { of Cushing's disease }(n=24)\end{array}$ & $\begin{array}{l}\text { Matched healthy controls } \\
\qquad(n=24)\end{array}$ & $P$-value \\
\hline Montgomery-Åsberg Depression Rating Scale (MADRS), mean (SD) & $6.37(5.59)$ & $1.42(1.79)$ & $<0.001^{a_{3} * * *}$ \\
\hline Beck Anxiety Inventory (BAI), mean (SD) & $28.48(5.79)$ & $24.33(3.16)$ & $0.010^{\mathrm{a}, *}$ \\
\hline Fear Questionnaire (FQ), mean (SD) & $25.52(17.00)$ & 13.92 (9.72) & $0.006^{\mathrm{b}, *}$ \\
\hline Social phobia subscale, mean (SD) & $12.70(7.77)$ & $7.79(4.82)$ & $0.012^{\mathrm{b}, *}$ \\
\hline Irritability scale (IS), mean (SD) & $12.13(8.91)$ & $8.54(6.02)$ & $0.176^{\mathrm{a}}$ \\
\hline Apathy scale (AS), mean (SD) & $|3.9|(6.50)$ & $8.13(3.80)$ & $0.001^{\mathrm{b}, * *}$ \\
\hline Cognitive failure questionnaire, mean (SD) & $37.39(16.55)$ & $28.58(9.10)$ & $0.028^{\mathrm{b}, *}$ \\
\hline
\end{tabular}

*P $<0.05$, uncorrected; $* * * 0.005$, Bonferroni corrected.

aMann-Whitney U-test.

${ }^{b}$ Independent sample t-test.

13 patients who were treated and those who were not $(t=0.396 ; p=0.696)$.

Analyses of between-group differences in RSFC with the DMN revealed aberrant connectivity with a small cluster located in the superior division of the left lateral occipital cortex ( $p<0.05$, TFCE corrected; Figure 3a). Examination of the individual $z$-scores showed positive connectivity of this area with the DMN in the patients and negative connectivity in the healthy control group (Figure $3 \mathrm{~b}$ ). Linear regression analyses with adjustment for age and education did not show a significant association between individual $z$-scores and the symptom scores of the behavioral measurements as well as the subscales of the CSI and the disease and remission duration in the patients with long-term remission of Cushing's disease. In addition, there was no difference in $z$-scores between patients with and without hydrocortisone treatment $(t=0.063 ; p=0.950)$. Contrasts testing the between-group differences in RSFC of the executive control network did not show any effects.

Omitting the voxel-wise gray matter covariate from the statistical model did not change the functional connectivity results as described in the previous section. This indicates that the altered RSFC within the three networks is unlikely to be related to macroscopic (ie, MRI observable) gray matter abnormalities.

\section{DISCUSSION}

We examined RSFC in patients with long-term remission of Cushing's disease and hypothesized to find aberrant RSFC of three networks of interest including the limbic network, the $\mathrm{DMN}$, and the executive control network in patients with long-term remission of Cushing's disease compared with matched healthy controls. Our hypothesis was confirmed for both the limbic network and the DMN, but not for the executive control network.
In patients we found an increase in RSFC between the limbic network and the subgenual ACC and an increase in RSFC between the DMN and a small cluster located in the superior division of the left lateral occipital cortex. Animal studies suggest that the subgenual ACC is a target site for the negative feedback effects of glucocorticoids on stress-induced HPA axis activity. Damage to this location leads to a diminished ability to regulate and inhibit HPA axis activity (Diorio et al, 1993). This has also been observed in humans, in whom behavioral studies showed that the subgenual ACC function is involved in emotion regulation (Bush et al, 2000; Phillips et al, 2003a; Quirk and Beer, 2006; Shin et al, 2000). The subgenual ACC exerts top-down inhibitory control over subcortical structures, most importantly the amygdala (Kim et al, 2011). Failing to exert this inhibitory control could lead to the disturbed emotion regulation seen in stress-related psychiatric disorders (Johnstone et al, 2007; Patel et al, 2012; Phillips et al, 2003b). Therefore, the subgenual ACC is the target site for some specific forms of treatment for stressrelated disorders. Deep brain stimulation of the subgenual ACC is currently used as a last resort treatment for treatment-resistant depression (Johansen-Berg et al, 2008; Lozano et al, 2008; Mayberg et al, 2005). Furthermore, subgenual ACC activity has been found to change under influence of fluoxetine use (Mayberg et al, 2000), an antidepressant that is used as a treatment for both PTSD and depression. These studies suggest that targeting subgenual ACC function is an effective treatment element for stress-related psychiatric disorders.

Our finding of increased RSFC connectivity between the limbic network and the subgenual ACC in patients with long-term remission of Cushing's disease suggest that these changes in coupling are established under the influence of hypercortisolism. This hypothesis is further supported by a study by Veer et al (2012) who found that RSFC connectivity between the amygdala (a key component of the limbic network) and the subgenual ACC is reactive to endogenous 

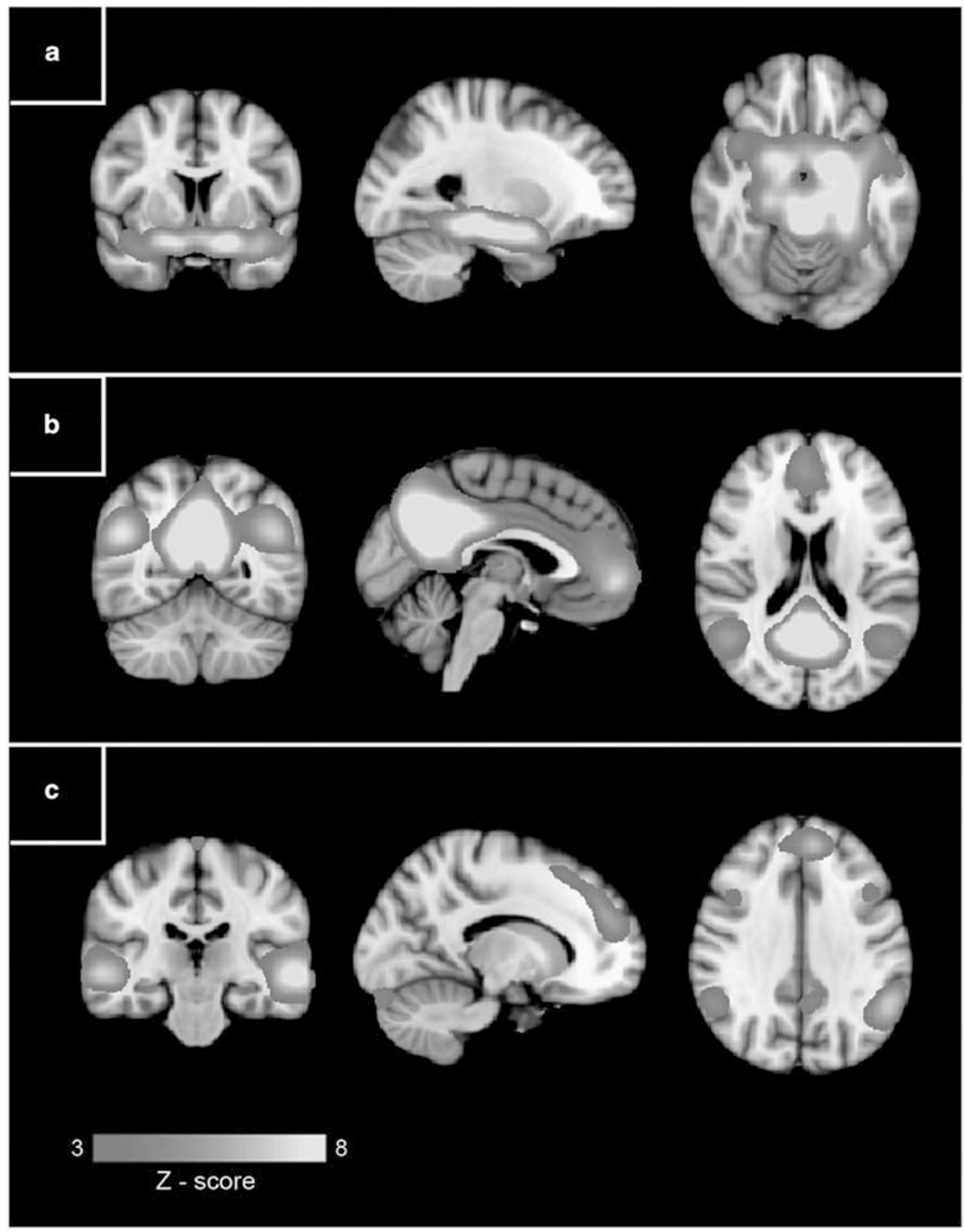

Figure I Resting-state networks of interest. (a) The limbic network. (b) The default mode network. (c) The executive control network. Images are z-statistics, overlaid on the MNI- I 52 I mm standard brain. The left hemisphere of the brain corresponds to the right side of the coronal and transversal images.

cortisol. In addition, our findings indicate that elevated RSFC between the subgenual ACC and key regions of the limbic network (like amygdala and insula) in depression (Connolly et al, 2013) and PTSD (Brown et al, 2014; Gilboa et al, 2004) could be an effect of exposure to high levels of cortisol often accompanying these stress-related disorders.

It is well known that optimal HPA axis reactivity is crucial to make correct adaptive responses in challenging situations but, once out of balance, can also be related to vulnerability or the emergence of psychiatric symptoms. We did not find an association of any of the behavioral measurements with the strength of the connectivity between the limbic network and the subgenual ACC. As our study was designed to test group differences, our study could have been underpowered to test for this type of associations. Therefore, it remains unknown whether these increases in connectivity reflect or underlie psychiatric symptoms, or more adaptive behavior. Recently, the latter has been suggested for increased RSFC between the hippocampus and the ventromedial prefrontal cortex in a group of trauma-exposed combat paramedics (Admon et al, 2013).

We also found an increase in RSFC in the patient group between the DMN and a small cluster located in the superior division of the left lateral occipital cortex. This area is situated adjacent to parietal regions that are part of the DMN. Therefore, an interpretation could be that the DMN of the patients recruits larger areas compared with healthy controls. This may indicate a less efficient use of the DMN in patients with long-term remission of Cushing's disease. 

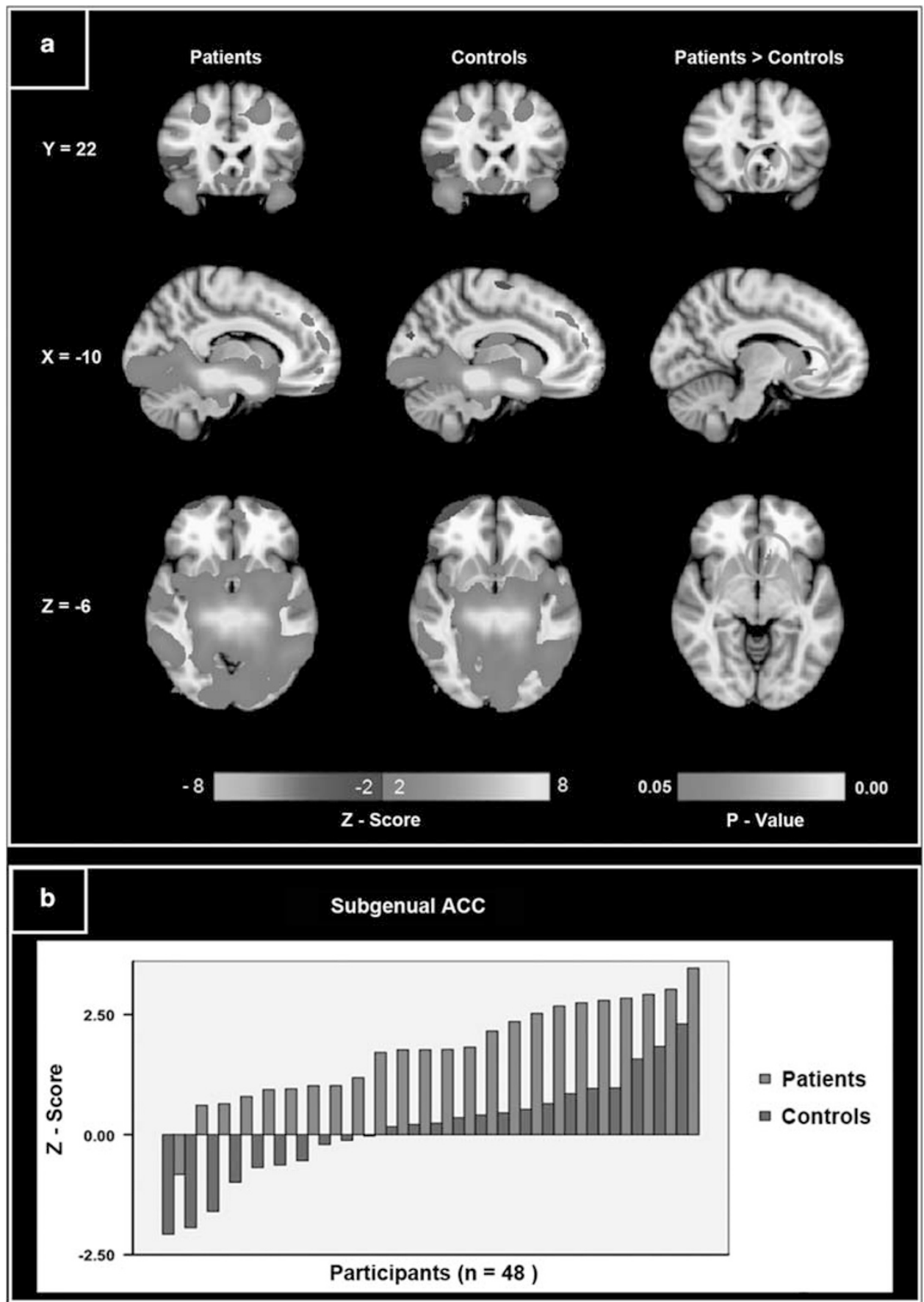

Figure 2 Resting-state functional connectivity of the limbic network. (a) Depicted here are the within-group mean z-scores and the between-group effect for the limbic network. Images representing the statistics are overlaid on the MNI- 152 I mm standard brain. The between-group effect is displayed as $p$-statistics, TFCE corrected for family-wise error $(p<0.05)$. The between-group effect shows increased RSFC between the limbic network and the subgenual ACC. (b) Distribution of the mean individual z-scores within the subgenual ACC. Depicted in red are the patients with long-term remission of Cushing's disease, depicted in blue are the matched healthy controls, and both are sorted from smallest to highest z-score. A full colour version of this figure is available at the Neuropsychopharmacology journal online.

Contrary to our expectations, the executive control network did not show differences in RSFC between the patients and controls. However, the observed cognitive impairment still present in the patients with Cushing's disease in remission was subtle, and the cognitive demands during resting state are low because of the absence of specific goal-oriented tasks. Consequently, differences in functional activity might only be expressed when demands on cognition are high (ie, during tasks). Task-related fMRI studies with a high cognitive load could be used to investigate this, as these tasks also show aberrant activity patterns in the executive control network in patients with depression and PTSD (Aizenstein et al, 2009; Daniels et al, 2010; Wang et al, 2008).

Limitations of our study consist of the cross-sectional design of our study that does not permit any causal 

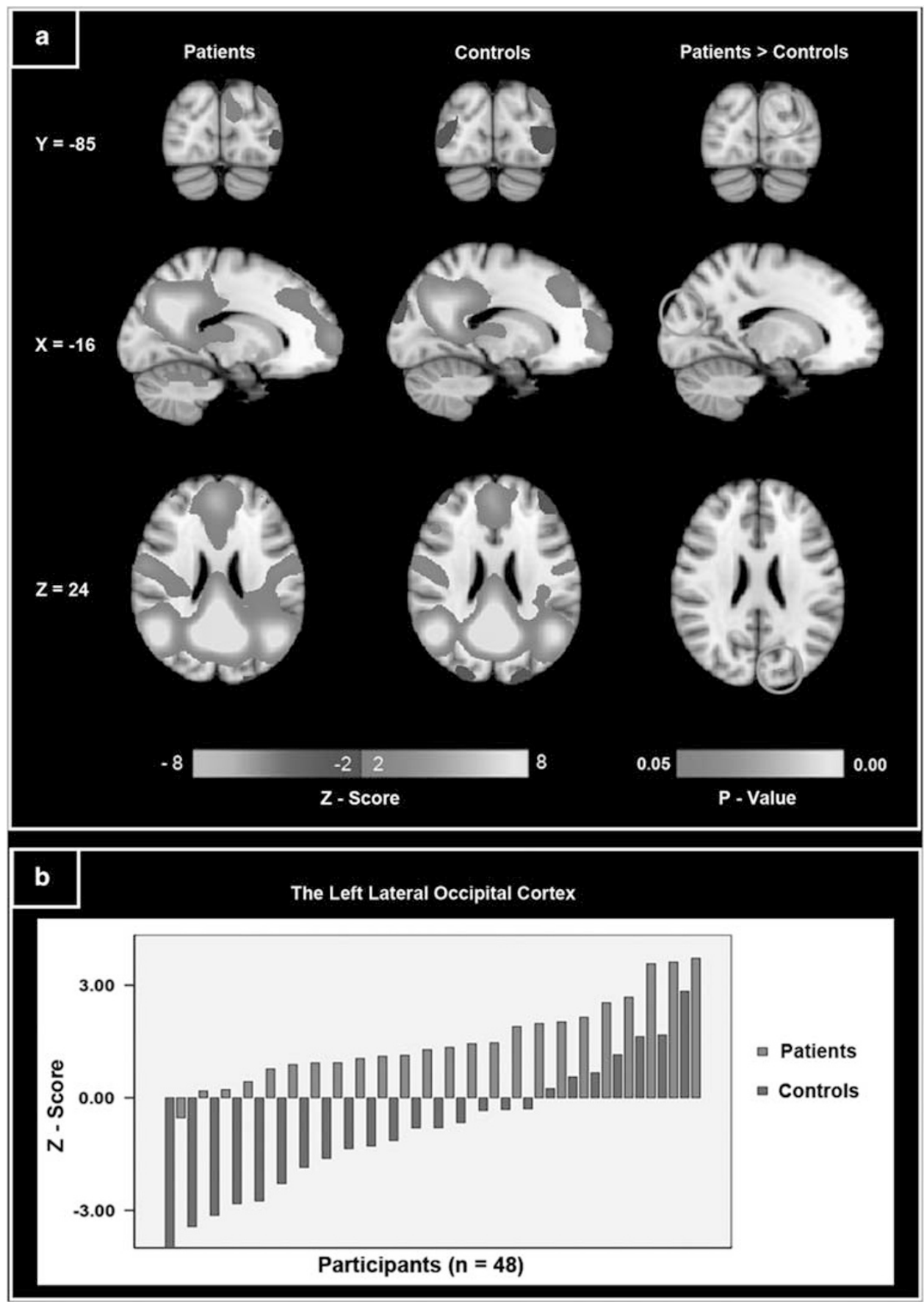

Figure 3 Resting-state functional connectivity of the default mode network. (a) Depicted here are the within-group mean z-scores and between-group effect for the default mode network. Images representing the statistics are overlaid on the MNI-I52 I mm standard brain. The between-group effect is displayed as $p$-statistics, TFCE corrected for family-wise error $(p<0.05)$. The between-group effect shows increased RSFC between the default mode network and a small cluster located in the superior division of the left lateral occipital cortex. (b) Distribution of the mean individual z-scores within the superior division of the left lateral occipital cortex. Depicted in red are the patients with long-term remission of Cushing's disease, depicted in blue are the matched healthy controls, and both are sorted from smallest to highest z-score.

conclusions to be drawn from the data. Therefore, we cannot rule out that the found between-group differences preceded the onset of the Cushing's disease. It is also possible that the difference in connectivity is an effect of the depressive symptomatology in the patient group that developed independent of the exposure to hypercortisolism. However, we did not find a correlation between depression severity and the strength of connectivity, making it less likely that the difference in connectivity purely reflects psychopathology. Another limitation is that we did not correct for the number of networks investigated in this study, thereby giving the study an explorative nature. Finally, we have not applied global signal regression that is known to induce negative connectivity between networks (Murphy et al, 2009). However, this also means our method is less sensitive toward detecting negative connectivity in the data. 
In summary, findings from neuroendocrine animal models for stress-related disorders using rodents with chronically elevated levels of corticosterone may have limited applicability to human brain function during challenges or resting state. Cushing's disease represents a unique model for investigating effects of prolonged hypercortisolism on the human brain. Earlier studies using Cushing's disease to investigate the long-term effects of hypercortisolism on the structure of the brain showed smaller gray matter volumes of the ACC, increased gray matter volumes of the cerebellum, and reduced structural connectivity to be rather persistent (Andela et al, 2013; van der Werff et al, 2014), whereas normalization of cortisol levels reversed hippocampal atrophy following treatment of Cushing's disease (Starkman et al, 1999). Our study is the first to suggest that exposure to hypercortisolism may lead to persistent changes in brain functional connectivity as well. Our finding of increased RSFC between the limbic network and the subgenual ACC is similar to the results of studies performed in patients with stress-related disorders, suggesting that these changes in RSFC in these stress-related disorders are established under the influence of increased levels of cortisol.

Future studies in the effects of stress or cortisol on RSFC should focus on connectivity between the limbic network (or specific key regions of this network) and the subgenual ACC in order to replicate this finding. This could also be achieved by conducting a targeted approach using the subgenual ACC as seed to investigate connectivity differences. Longitudinally designed studies should be performed to investigate the causal effects of hypercortisolism on RSFC. In addition, taskrelated fMRI paradigms could contribute to investigate changes in brain activity during specific demands.

\section{FUNDING AND DISCLOSURE}

Over the past 3 years, NJA Van der Wee has received compensation for consultancy from Servier, manufacturer of agomelatine, and Pfizer, manufacturer of gabapentin. The authors declare no conflict of interest.

\section{ACKNOWLEDGMENTS}

SJA Van der Werff was supported through the Netherlands Organization for Scientific Research-National Initiative Brain and Cognition project (NWO-NIHC, project no. 056-25-010). NR Biermasz was supported through the Netherlands Organization for Scientific Research (NWOVENI, project no. 016136125). SARB Rombouts was supported through the Netherlands Organizaton for Scientific Research (NWO-VICI, project no. 016130677).

\section{REFERENCES}

Admon R, Leykin D, Lubin G, Engert V, Andrews J, Pruessner J et al (2013). Stress-induced reduction in hippocampal volume and connectivity with the ventromedial prefrontal cortex are related to maladaptive responses to stressful military service. Hum Brain Mapp 34: 2808-2816.

Admon R, Lubin G, Stern O, Rosenberg K, Sela L, Ben-Ami H et al (2009). Human vulnerability to stress depends on amygdala's predisposition and hippocampal plasticity. Proc Natl Acad Sci USA 106: 14120-14125.
Aizenstein HJ, Butters MA, Wu M, Mazurkewicz LM, Stenger VA, Gianaros PJ et al (2009). Altered functioning of the executive control circuit in late-life depression: episodic and persistent phenomena. Am J Geriatr Psychiatry 17: 30-42.

Andela CD, van der Werff SJ, Pannekoek JN, van den Berg SM, Meijer OC, van Buchem MA et al (2013). Smaller grey matter volumes in the anterior cingulate cortex and greater cerebellar volumes in patients with long-term remission of Cushing's disease: a case-control study. Eur J Endocrinol 169: 811-819.

Beck AT, Epstein N, Brown G, Steer RA (1988). An inventory for measuring clinical anxiety: psychometric properties. J Consult Clin Psychol 56: 893-897.

Beckmann CF, DeLuca M, Devlin JT, Smith SM (2005). Investigations into resting-state connectivity using independent component analysis. Philos Trans R Soc Lond B Biol Sci 360: 1001-1013.

Beckmann CF, Smith SM (2004). Probabilistic independent component analysis for functional magnetic resonance imaging. IEEE Trans Med Imaging 23: 137-152.

Bluhm R, Williamson P, Lanius R, Theberge J, Densmore M, Bartha $\mathrm{R}$ et al (2009a). Resting state default-mode network connectivity in early depression using a seed region-of-interest analysis: decreased connectivity with caudate nucleus. Psychiatry Clin Neurosci 63: 754-761.

Bluhm RL, Williamson PC, Osuch EA, Frewen PA, Stevens TK, Boksman $\mathrm{K}$ et al (2009b). Alterations in default network connectivity in posttraumatic stress disorder related to early-life trauma. J Psychiatry Neurosci 34: 187-194.

Boldrini M, Santiago AN, Hen R, Dwork AJ, Rosoklija GB, Tamir H et al (2013). Hippocampal granule neuron number and dentate gyrus volume in antidepressant-treated and untreated major depression. Neuropsychopharmacology 38: 1068-1077.

Bourdeau I, Bard C, Noel B, Leclerc I, Cordeau MP, Belair M et al (2002). Loss of brain volume in endogenous Cushing's syndrome and its reversibility after correction of hypercortisolism. J Clin Endocrinol Metab 87: 1949-1954.

Bremner JD, Vythilingam M, Vermetten E, Southwick SM, McGlashan T, Nazeer A et al (2003). MRI and PET study of deficits in hippocampal structure and function in women with childhood sexual abuse and posttraumatic stress disorder. Am J Psychiatry 160: 924-932.

Broadbent DE, Cooper PF, FitzGerald P, Parkes KR (1982). The Cognitive Failures Questionnaire (CFQ) and its correlates. Br J Clin Psychol 21(Pt 1): 1-16.

Brown VM, LaBar KS, Haswell CC, Gold AL, McCarthy G, Morey RA (2014). Altered resting-state functional connectivity of basolateral and centromedial amygdala complexes in posttraumatic stress disorder. Neuropsychopharmacology 39: 351-359.

Bush G, Luu P, Posner MI (2000). Cognitive and emotional influences in anterior cingulate cortex. Trends Cogn Sci 4: 215-222.

Campbell S, Marriott M, Nahmias C, MacQueen GM (2004). Lower hippocampal volume in patients suffering from depression: a meta-analysis. Am J Psychiatry 161: 598-607.

Carroll, Curtis GC, Mendels J (1976). Neuroendocrine regulation in depression. I. Limbic system-adrenocortical dysfunction. Arch Gen Psychiatry 33: 1039-1044.

Carroll BJ, Cassidy F, Naftolowitz D, Tatham NE, Wilson WH, Iranmanesh A et al (2007). Pathophysiology of hypercortisolism in depression. Acta Psychiatr Scand Suppl 433: 90-103.

Chatterjee A, Anderson KE, Moskowitz CB, Hauser WA, Marder KS (2005). A comparison of self-report and caregiver assessment of depression, apathy, and irritability in Huntington's disease. J Neuropsychiatry Clin Neurosci 17: 378-383.

Colla M, Kronenberg G, Deuschle M, Meichel K, Hagen T, Bohrer $\mathrm{M}$ et al (2007). Hippocampal volume reduction and HPAsystem activity in major depression. J Psychiatr Res 41: 553-560.

Connolly CG, Wu J, Ho TC, Hoeft F, Wolkowitz O, Eisendrath S et al (2013). Resting-state functional connectivity of subgenual 
anterior cingulate cortex in depressed adolescents. Biol Psychiatry 74: 898-907.

Daniels JK, McFarlane AC, Bluhm RL, Moores KA, Clark CR, Shaw ME et al (2010). Switching between executive and default mode networks in posttraumatic stress disorder: alterations in functional connectivity. J Psychiatry Neurosci 35: 258-266.

Darcet F, Mendez-David I, Tritschler L, Gardier AM, Guilloux JP, David DJ (2014). Learning and memory impairments in a neuroendocrine mouse model of anxiety/depression. Front Behav Neurosci 8: 136.

David DJ, Samuels BA, Rainer Q, Wang JW, Marsteller D, Mendez I et al (2009). Neurogenesis-dependent and -independent effects of fluoxetine in an animal model of anxiety/depression. Neuron 62: 479-493.

Diorio D, Viau V, Meaney MJ (1993). The role of the medial prefrontal cortex (cingulate gyrus) in the regulation of hypothalamicpituitary-adrenal responses to stress. J Neurosci 13: 3839-3847.

Dorn LD, Cerrone P (2000). Cognitive function in patients with Cushing syndrome: a longitudinal perspective. Clin Nurs Res 9: 420-440.

Filippini N, MacIntosh BJ, Hough MG, Goodwin GM, Frisoni GB, Smith SM et al (2009). Distinct patterns of brain activity in young carriers of the APOE-epsilon4 allele. Proc Natl Acad Sci USA 106: $7209-7214$.

Forget H, Lacroix A, Cohen H (2002). Persistent cognitive impairment following surgical treatment of Cushing's syndrome. Psychoneuroendocrinology 27: 367-383.

Fox MD, Snyder AZ, Vincent JL, Corbetta M, Van Essen DC, Raichle ME (2005). The human brain is intrinsically organized into dynamic, anticorrelated functional networks. Proc Natl Acad Sci USA 102: 9673-9678.

Friedman MJ, Jalowiec J, McHugo G, Wang S, McDonagh A (2007). Adult sexual abuse is associated with elevated neurohormone levels among women with PTSD due to childhood sexual abuse. J Trauma Stress 20: 611-617.

Gilboa A, Shalev AY, Laor L, Lester H, Louzoun Y, Chisin R et al (2004). Functional connectivity of the prefrontal cortex and the amygdala in posttraumatic stress disorder. Biol Psychiatry 55: 263-272.

Gurvits TV, Shenton ME, Hokama H, Ohta H, Lasko NB, Gilbertson MW et al (1996). Magnetic resonance imaging study of hippocampal volume in chronic, combat-related posttraumatic stress disorder. Biol Psychiatry 40: 1091-1099.

Hyvarinen A (1999). Fast and robust fixed-point algorithms for independent component analysis. IEEE Trans Neural Netw 10: 626-634.

Inslicht SS, Marmar CR, Neylan TC, Metzler TJ, Hart SL, Otte C et al (2006). Increased cortisol in women with intimate partner violence-related posttraumatic stress disorder. Ann NY Acad Sci 1071: 428-429.

Janes AC, Nickerson LD, Frederick Bde B, Kaufman MJ (2012). Prefrontal and limbic resting state brain network functional connectivity differs between nicotine-dependent smokers and non-smoking controls. Drug Alcohol Depend 125: 252-259.

Jin C, Qi R, Yin Y, Hu X, Duan L, Xu Q et al (2014). Abnormalities in whole-brain functional connectivity observed in treatmentnaive post-traumatic stress disorder patients following an earthquake. Psychol Med 44: 1927-1936.

Johansen-Berg H, Gutman DA, Behrens TE, Matthews PM, Rushworth MF, Katz E et al (2008). Anatomical connectivity of the subgenual cingulate region targeted with deep brain stimulation for treatment-resistant depression. Cereb Cortex 18: 1374-1383.

Johnstone T, van Reekum CM, Urry HL, Kalin NH, Davidson RJ (2007). Failure to regulate: counterproductive recruitment of top-down prefrontal-subcortical circuitry in major depression. J Neurosci 27: 8877-8884.

Kasai K, Yamasue H, Gilbertson MW, Shenton ME, Rauch SL, Pitman RK (2008). Evidence for acquired pregenual anterior cingulate gray matter loss from a twin study of combat-related posttraumatic stress disorder. Biol Psychiatry 63: 550-556.

Kim MJ, Loucks RA, Palmer AL, Brown AC, Solomon KM, Marchante AN et al (2011). The structural and functional connectivity of the amygdala: from normal emotion to pathological anxiety. Behav Brain Res 223: 403-410.

Kitayama N, Vaccarino V, Kutner M, Weiss P, Bremner JD (2005). Magnetic resonance imaging (MRI) measurement of hippocampal volume in posttraumatic stress disorder: a meta-analysis. J Affect Disord 88: 79-86.

Laird AR, Fox PM, Eickhoff SB, Turner JA, Ray KL, McKay DR et al (2011). Behavioral interpretations of intrinsic connectivity networks. J Cogn Neurosci 23: 4022-4037.

Lozano AM, Mayberg HS, Giacobbe P, Hamani C, Craddock RC, Kennedy SH (2008). Subcallosal cingulate gyrus deep brain stimulation for treatment-resistant depression. Biol Psychiatry 64: 461-467.

Maclean PD (1952). Some psychiatric implications of physiological studies on frontotemporal portion of limbic system (visceral brain). Electroencephalogr Clin Neurophysiol 4: 407-418.

Marks IM, Mathews AM (1979). Brief standard self-rating for phobic patients. Behav Res Ther 17: 263-267.

Mayberg HS, Brannan SK, Tekell JL, Silva JA, Mahurin RK, McGinnis S et al (2000). Regional metabolic effects of fluoxetine in major depression: serial changes and relationship to clinical response. Biol Psychiatry 48: 830-843.

Mayberg HS, Lozano AM, Voon V, McNeely HE, Seminowicz D, Hamani C et al (2005). Deep brain stimulation for treatmentresistant depression. Neuron 45: 651-660.

Merke DP, Giedd JN, Keil MF, Mehlinger SL, Wiggs EA, Holzer S et al (2005). Children experience cognitive decline despite reversal of brain atrophy one year after resolution of Cushing syndrome. J Clin Endocrinol Metab 90: 2531-2536.

Montgomery SA, Asberg M (1979). A new depression scale designed to be sensitive to change. Br J Psychiatry 134: 382-389.

Murphy K, Birn RM, Handwerker DA, Jones TB, Bandettini PA (2009). The impact of global signal regression on resting state correlations: are anti-correlated networks introduced? Neuroimage 44: 893-905.

Nordanskog P, Dahlstrand U, Larsson MR, Larsson EM, Knutsson L, Johanson A (2010). Increase in hippocampal volume after electroconvulsive therapy in patients with depression: a volumetric magnetic resonance imaging study. J Ect 26: 62-67.

Oakes TR, Fox AS, Johnstone T, Chung MK, Kalin N, Davidson RJ (2007). Integrating VBM into the general linear model with voxelwise anatomical covariates. Neuroimage 34: 500-508.

Patel R, Spreng RN, Shin LM, Girard TA (2012). Neurocircuitry models of posttraumatic stress disorder and beyond: a metaanalysis of functional neuroimaging studies. Neurosci Biobehav Rev 36: 2130-2142.

Phillips ML, Drevets WC, Rauch SL, Lane R (2003a). Neurobiology of emotion perception I: The neural basis of normal emotion perception. Biol Psychiatry 54: 504-514.

Phillips ML, Drevets WC, Rauch SL, Lane R (2003b). Neurobiology of emotion perception II: implications for major psychiatric disorders. Biol Psychiatry 54: 515-528.

Quirk GJ, Beer JS (2006). Prefrontal involvement in the regulation of emotion: convergence of rat and human studies. Curr Opin Neurobiol 16: 723-727.

Raichle ME, MacLeod AM, Snyder AZ, Powers WJ, Gusnard DA, Shulman GL (2001). A default mode of brain function. Proc Natl Acad Sci USA 98: 676-682.

Resmini E, Santos A, Gomez-Anson B, Vives Y, Pires P, Crespo I et al (2012). Verbal and visual memory performance and hippocampal volumes, measured by 3 -Tesla magnetic resonance imaging, in patients with Cushing's syndrome. J Clin Endocrinol Metab 97: 663-671. 
Rush AJ, Gullion CM, Basco MR, Jarrett RB, Trivedi MH (1996). The Inventory of Depressive Symptomatology (IDS): psychometric properties. Psychol Med 26: 477-486.

Schlechte JA, Sherman B, Pfohl B (1986). A comparison of adrenal cortical function in patients with depressive illness and Cushing's disease. Horm Res 23: 1-8.

Seeley WW, Menon V, Schatzberg AF, Keller J, Glover GH, Kenna $\mathrm{H}$ et al (2007). Dissociable intrinsic connectivity networks for salience processing and executive control. J Neurosc 27: 2349-2356.

Shin LM, Dougherty DD, Orr SP, Pitman RK, Lasko M, Macklin ML et al (2000). Activation of anterior paralimbic structures during guilt-related script-driven imagery. Biol Psychiatry 48: 43-50.

Shin LM, Liberzon I (2010). The neurocircuitry of fear, stress, and anxiety disorders. Neuropsychopharmacology 35: 169-191.

Simmons NE, Do HM, Lipper MH, Laws ER Jr. (2000). Cerebral atrophy in Cushing's disease. Surg Neurol 53: 72-76.

Smith SM, Fox PT, Miller KL, Glahn DC, Fox PM, Mackay CE et al (2009a). Correspondence of the brain's functional architecture during activation and rest. Proc Natl Acad Sci USA 106: 13040-13045.

Smith SM, Jenkinson M, Woolrich MW, Beckmann CF, Behrens TE, Johansen-Berg $\mathrm{H}$ et al (2004). Advances in functional and structural MR image analysis and implementation as FSL. Neuroimage 23(Suppl 1): S208-S219.

Smith SM, Nichols TE (2009b). Threshold-free cluster enhancement: addressing problems of smoothing, threshold dependence and localisation in cluster inference. Neuroimage 44: 83-98.

Sonino N, Boscaro M, Fallo F, Fava GA (2000). A clinical index for rating severity in Cushing's syndrome. Psychother Psychosom 69: 216-220.

Sonino N, Fava GA (2001). Psychiatric disorders associated with Cushing's syndrome. Epidemiology, pathophysiology and treatment. CNS Drugs 15: 361-373.

Starkman MN, Gebarski SS, Berent S, Schteingart DE (1992). Hippocampal formation volume, memory dysfunction, and cortisol levels in patients with Cushing's syndrome. Biol Psychiatry 32: 756-765.

Starkman MN, Giordani B, Gebarski SS, Berent S, Schork MA, Schteingart DE (1999). Decrease in cortisol reverses human hippocampal atrophy following treatment of Cushing's disease. Biol Psychiatry 46: 1595-1602.

Starkstein SE, Petracca G, Chemerinski E, Kremer J (2001). Syndromic validity of apathy in Alzheimer's disease. Am J Psychiatry 158: 872-877.

Steudte S, Kolassa IT, Stalder T, Pfeiffer A, Kirschbaum C, Elbert T (2011). Increased cortisol concentrations in hair of severely traumatized Ugandan individuals with PTSD. Psychoneuroendocrinology 36: 1193-1200.

Tendolkar I, van Beek M, van Oostrom I, Mulder M, Janzing J, Voshaar RO et al (2013). Electroconvulsive therapy increases hippocampal and amygdala volume in therapy refractory depression: a longitudinal pilot study. Psychiatry Res 214: 197-203.

Thomaes K, Dorrepaal E, Draijer N, de Ruiter MB, van Balkom AJ, Smit JH et al (2010). Reduced anterior cingulate and orbitofrontal volumes in child abuse-related complex PTSD. J Clin Psychiatry 71: 1636-1644.

Tiemensma J, Biermasz NR, Middelkoop HA, van der Mast RC, Romijn JA, Pereira AM (2010a). Increased prevalence of psychopathology and maladaptive personality traits after longterm cure of Cushing's disease. J Clin Endocrinol Metab 95: E129-E141.

Tiemensma J, Kaptein AA, Pereira AM, Smit JW, Romijn JA, Biermasz NR (2011). Negative illness perceptions are associated with impaired quality of life in patients after long-term remission of Cushing's syndrome. Eur J Endocrinol 165: 527-535.

Tiemensma J, Kokshoorn NE, Biermasz NR, Keijser BJ, Wassenaar MJ, Middelkoop HA et al (2010b). Subtle cognitive impairments in patients with long-term cure of Cushing's disease. J Clin Endocrinol Metab 95: 2699-2714.

van der Werff SJ, Andela CD, Nienke Pannekoek J, Meijer OC, van Buchem MA, Rombouts SA et al (2014). Widespread reductions of white matter integrity in patients with long-term remission of Cushing's disease. Neuroimage Clin 4: 659-667.

Veer IM, Beckmann CF, van Tol M-J, Ferrarini L, Milles J, Veltman DJ et al (2010). Whole brain resting-state analysis reveals decreased functional connectivity in major depression. Front Syst Neurosci 4: 41(doi: 10.3389/fnsys.2010.00041).

Veer IM, Oei NY, Spinhoven P, van Buchem MA, Elzinga BM, Rombouts SA (2012). Endogenous cortisol is associated with functional connectivity between the amygdala and medial prefrontal cortex. Psychoneuroendocrinology 37: 1039-1047.

Vermetten E, Vythilingam M, Southwick SM, Charney DS, Bremner JD (2003). Long-term treatment with paroxetine increases verbal declarative memory and hippocampal volume in posttraumatic stress disorder. Biological Psychiatry 54: 693-702.

Videbech P, Ravnkilde B (2004). Hippocampal volume and depression: a meta-analysis of MRI studies. Am J Psychiatry 161: 1957-1966.

Wang L, LaBar KS, Smoski M, Rosenthal MZ, Dolcos F, Lynch TR et al (2008). Prefrontal mechanisms for executive control over emotional distraction are altered in major depression. Psychiatry Res 163: 143-155.

Yamasue H, Kasai K, Iwanami A, Ohtani T, Yamada H, Abe O et al (2003). Voxel-based analysis of MRI reveals anterior cingulate gray-matter volume reduction in posttraumatic stress disorder due to terrorism. Proc Natl Acad Sci USA 100: 9039-9043.

Young EA, Breslau N (2004). Saliva cortisol in posttraumatic stress disorder: a community epidemiologic study. Biol Psychiatry 56: 205-209.

Zhu X, Wang X, Xiao J, Liao J, Zhong M, Wang W et al (2012). Evidence of a dissociation pattern in resting-state default mode network connectivity in first-episode, treatment-naive major depression patients. Biol Psychiatry 71: 611-617. 